\title{
EFECTO DE LA URBANIZACIÓN EN EL BALANCE DE ENERGÍA SUPERFICIAL DE UN BOSQUE DE NIEBLA AL ESTE DE MÉXICO
}

\author{
Carlo A. DOMÍNGUEZ-EUSEBIO ${ }^{1}$, Adalberto TEJEDA-MARTÍNEZ², \\ Yareni PERRONI ${ }^{1}$ \\ ${ }^{1}$ Instituto de Biotecnología y Ecología Aplicada, Universidad Veracruzana, México. \\ ${ }^{2}$ Grupo de Climatología Aplicada, Universidad Veracruzana, México. \\ cardomingueze@gmail.com, atejeda.martinez@gmail.com, yperroni@uv.mx
}

\section{RESUMEN}

Características de la superficie como rugosidad, cobertura y capacidad térmica, condicionan el espesor de la capa límite superficial atmosférica y los procesos que ocurren en ella. Sin embargo, poco se sabe de las modificaciones en esos procesos ante cambios en la superficie de ecosistemas naturales. El objetivo de este estudio fue obtener una primera caracterización del efecto de la urbanización en el balance energético del bosque de niebla, ecosistema común en montañas tropicales. Se utilizó el método de covarianza turbulenta en dos estaciones micrometeorológicas: una en un bosque urbano (Parque Ecológico Cerro de Macuiltépetl: 19³2'51"N, 9655'122"W, $1580 \mathrm{msnm}$ ), y otra en un bosque natural (El Riscal: $19^{\circ} 28^{\prime} 54^{\prime \prime} \mathrm{N}, 96^{\circ} 59^{\prime} 44^{\prime \prime} \mathrm{W}, 1570$ msnm). Los resultados indican que durante el día, en la temporada seca (diciembre 2014-mayo 2015), la radiación neta media fue mayor en el bosque urbano (402,77 vs. $\left.367,67 \mathrm{~W} / \mathrm{m}^{2}\right)$, al igual que el flujo de calor sensible $\left(185,36 \mathrm{~W} / \mathrm{m}^{2} v s .168,86 \mathrm{~W} / \mathrm{m}^{2}\right)$, la temperatura $\left(18,17\right.$ vs. $\left.17,74^{\circ} \mathrm{C}\right)$, el flujo de calor latente $\left(119,37\right.$ vs. $\left.94,52 \mathrm{~W} / \mathrm{m}^{2}\right)$ y la evaporación (1,63 vs. 1,39 mm). El bosque natural presentó mayor calor almacenado en la superficie $\left(104,47 v s .98,04 \mathrm{~W} / \mathrm{m}^{2}\right)$. Estas diferencias podrían ser efecto de la urbanización en este ecosistema.

Palabras clave: flujos de energía, bosque de niebla, urbanización, covarianza turbulenta.

\begin{abstract}
The surface characteristics such as roughness, coverage and heat capacity determines the thickness of the atmospheric surface boundary layer and the processes occurring therein. However, the interaction of these systems in relation to changes in the surface of natural ecosystems has been little studied. In this way, the objective of this study was to conduct a first characterization of the effect of urbanization on the energy balance of cloud forest, a common ecosystem in tropical mountains. The eddy covariance method was used in two micrometeorological stations: in an urban forest (Parque Ecológico Cerro de Macuiltépetl: 19³2'51"N, 96 55'12"W, 1580 masl) and a natural forest (El Riscal: 19²8'54"N, 9659'44"W, 1570 masl). On the day, during the dry season (December 2014-May 2015), the average net radiation was higher in the urban forest $\left(402.77 v s .367 .67 \mathrm{~W} / \mathrm{m}^{2}\right)$, the sensible heat flux $\left(185.36 \mathrm{~W} / \mathrm{m}^{2} \mathrm{vs}\right.$. $\left.168.86 \mathrm{~W} / \mathrm{m}^{2}\right)$, temperature $\left(18.17 \mathrm{vs} .17 .74^{\circ} \mathrm{C}\right)$, the latent heat flux $(119.37 \mathrm{vs.} 94.52$
\end{abstract}


$\mathrm{W} / \mathrm{m}^{2}$ ) and evapotranspiration (1.63 vs. $\left.1.39 \mathrm{~mm}\right)$. The natural forest had higher heat stored in the surface $\left(104.47 v s .98 .04 \mathrm{~W} / \mathrm{m}^{2}\right)$. These differences could be the effect of urbanization in this ecosystem.

Key words: Energy flux, mountain tropical forest, eddy covariance, urbanization.

\section{INTRODUCCIÓN}

El tipo de superficie (rugosidad, cobertura, capacidad térmica, etcétera) juega una papel importante en el desarrollo de la capa limite superficial atmosférica, así como en los patrones de interacción entre la superficie y la atmósfera (Oke, 2002, Foken \& Nappo, 2008). Por esto, el cambio de uso de cobertura de suelo puede provocar alteraciones físicas en el ambiente.

La urbanización es uno de los cambios de uso de suelo con mayor tasa de crecimiento. Actualmente más de la mitad de la población humana se encuentra en ciudades (54\%; UN, 2014). En general, las grandes ciudades modifican el clima local de diferentes maneras: disminuye la radiación solar por la contaminación atmosférica, aumenta de temperatura en relación con sus alrededores, es menor la humedad relativa citadina, y es mayor la precipitación en el centro urbano al igual que la turbulencia del aire (Schönwiese, 1994; Oke et al., 1999).

La urbanización provoca una modificación en los flujos verticales de energía, en particular los de calor sensible y latente, y por ende en el balance energético superficial. Este balance cuantifica la cantidad de energía solar que es destinada para calentar el aire y evaporar agua, así como la almacenada y utilizada por la superficie y seres vivos (Oke, 2002, Foken \& Nappo, 2008).

Existen diversos estudios que muestran el efecto de la urbe en el balance de energía en diferentes tipos de ciudades y de ecosistemas, como por ejemplo los de Christen \& Vogt, 1983; Cleugh \& Oke, 1986; Barradas et al., 1999; Tejeda-Mártinez y Jáuregui-Ostos, 2005; Tejeda-Martínez et al., 2016 y Moreno et al., 2012. No obstante, los trabajos relacionados con el bosque de niebla se centran en el balance de energía sin considerar efectos de cambios de cobertura (e.g. Giambelluca et al, 2009), o en el efecto que tiene este cambio en procesos del suelo y/o vegetación (e.g. Campos, 2006).

El bosque de niebla es un ecosistema montañoso que presenta una alta biodiversidad vegetal y animal, además de que aporta diversos servicios ambientales (Wi1liam-Linera, 2012). La principal característica de estos ecosistemas es la frecuencia de niebla en la que se ven inmersos, lo cual ocasiona una entrada adicional de agua al ecosistema con consecuencias en la hidrología y propiedades del suelo (Standmuller, 1987; Bubb et al, 2004). Este ecosistema único en su tipo, sufre una amenaza importante por el cambio de uso de suelo para fines urbanos, agropecuarios e industriales. En México, el bosque de niebla abarca sólo el 1\% de la superficie total del país, siendo el ecosistema más biodiverso por unidad de área (William-Linera, 2012).

Por todo lo anterior, el objetivo de este trabajo es conocer cómo afecta la urbanización en la distribución de la energía dentro del ecosistema bosque de niebla. 


\section{MÉTODOS}

\subsection{Sitios de estudio}

El estudio se realizó en el Parque Ecológico Cerro del Macuiltépetl (19³2'51,26"N y 965 $55^{\prime} 12,66^{\prime \prime} \mathrm{W}$ a 1580 msnm con un área aproximada de 30 hectáreas), que presenta vegetación típica del bosque de niebla como liquidámbar, cedros, hayas, fresnos y algunos cítricos (Williams-Linera, 2002). Este "bosque urbano" es una reserva natural protegida desde 1980 (Aguilar, 1996) con la peculiaridad de ser un parche de bosque de niebla completamente rodeado por la ciudad de Xalapa, Veracruz, México (19 $30^{\prime} 01,03^{\prime \prime}$ a $19^{\circ} 36^{\prime} 05,87^{\prime \prime} \mathrm{N}$ y $96^{\circ} 54^{\prime} 14,20^{\prime \prime}$ a $\left.97^{\circ} 02^{\prime} 43,11^{\prime \prime W}\right)$. Esta ciudad cuenta con aproximadamente 425 mil habitantes, siendo la urbe número 39 de las más pobladas de México (INEGI, 2010). Presenta una temperatura media anual de $19,2^{\circ} \mathrm{C}$ y un precipitación anual de 1435,8 mm (SMN, 2016).

Para comparar el efecto de la urbanización con un punto con mínima perturbación antropogénica, se seleccionó el rancho El Riscal, Coatepec, Veracruz, México $\left(19^{\circ} 28^{\prime} 54,04^{\prime \prime} \mathrm{N}, 96^{\circ} 59^{\prime} 44,80^{\prime \prime} \mathrm{W}\right)$, ubicado a $10 \mathrm{~km}$ de distancia en línea recta al Oeste-Suroeste de la ciudad de Xalapa, a una altitud media de $1570 \mathrm{msnm}$. Se considera como un "bosque natural" ya que es un área boscosa rodeada por más vegetación. Se eligió este sitio por coincidir en altitud con el bosque urbano y porque se encuentra a corta distancia de aquél. Así, se espera que las diferencias observadas en las mediciones de balance de energía se deban principalmente al efecto de la urbe y no por la afectación de fenómenos meteorológicos diferentes en cada sitio (Fig. 1).

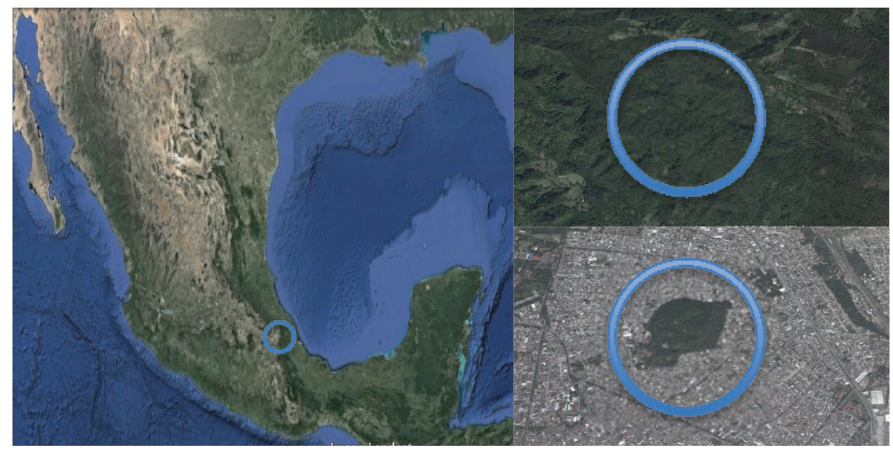

Fig. 1. Sitios de estudio, a)El Riscal, b)El Macuiltépetl. Fuente: Google Earth.

\subsection{Instrumentos y datos.}

En ambos sitios se instaló una torre de $18 \mathrm{~m}$ de altura con instrumentos útiles para conocer los flujos de energía superficial y el balance de radiación. Cada una cuenta con un anemómetro sónico CSAT-3D (Campbell Scientific) para medir la dirección e intensidad del viento en tres direcciones $(U, V, W)$; un higrómetro de kriptón $\mathrm{KH} 2 \mathrm{O}$ (Campbell Scientific) para la humedad absoluta (HA); un termopar FW05 (Campbell Scietific) para la temperatura del aire (Ta), y un radiómetro neto NR01 (Campbell Scientific) con el que se obtuvieron las cuatro componentes de radiación: incidente y reflejada de onda corta ( $\mathrm{Rc} \downarrow$ y $\mathrm{Rc} \uparrow$ ), e incidente y emitida de onda larga (Rl $\downarrow$ y Rl $\uparrow$ ). 
Estos sensores fueron programados a $10 \mathrm{~Hz}$ para utilizar la técnica de covariaza turbulenta (Eddy covariance; Burba, 2013; Baldocchi, 1988) en la evaluación de los flujos verticales turbulentos de calor sensible y latente. Los datos fueron procesados y almacenados en un micrologger CR3000 (Campbell Scientific) configurado para almacenar datos cada 15 minutos. Esta evaluación fue realizada de diciembre 2014 a mayo 2015, correspondiente a la temporada seca. Cabe mencionar que sólo se analizaron días despejados ya que durante eventos de lluvia, por las características de los instrumentos, hay pérdida de datos.

El balance de energía superficial se obtuvo mediante la ecuación (Monteith \& Unsworth, 2008; Foken \& Nappo, 2008; Oke 2002):

$$
Q^{*}=Q h+Q e+Q s
$$

donde $Q^{*}$ es la radiación neta obtenida mediante la diferencia entre la radiación de onda corta y larga incidente y saliente $(\mathrm{Rc} \downarrow+\mathrm{Rl} \downarrow-\mathrm{Rc} \uparrow-\mathrm{Rl} \uparrow$ ), $Q h$ en el flujo turbulento vertical de calor sensible, $Q e$ el flujo turbulento vertical de calor latente, $\mathrm{y}$ $Q s$ es el flujo de energía en la superficie (la suma del calor almacenado en el suelo y en la vegetación). Cuando $Q^{*}$ presenta valores negativos, el sistema pierde energía por radiación. Si $Q h, Q e$ o $Q s$ presentan valores negativos, significa que el sistema está ganando energía de los alrededores.

El flujo de calor sensible $(Q h)$ fue obtenido mediante la ecuación:

$$
Q h=\rho C p \overline{W^{\prime} T a^{\prime}}
$$

con $\rho$ la densidad del aire $\left(1.02 \mathrm{~kg} \mathrm{~m}^{-3}\right.$, en promedio para las condiciones de estos sitios), $C p$ el calor específico del aire a presión constante $\left(1012 \mathrm{JKg}^{-1} \mathrm{~K}^{-1}\right)$ y $\overline{W^{\prime} T a^{\prime}}$ la covarianza entre la velocidad del viento vertical y la temperatura del aire. El flujo de calor latente $(Q e)$ fue obtenido mediante la ecuación:

$$
Q e=\lambda \overline{W^{\prime} H A^{\prime}}
$$

donde $\lambda$ es el calor latente de evaporación $\left(2500 \mathrm{~kJ} \mathrm{~kg}^{-1}\right)$ y $\overline{W^{\prime} H A^{\prime}}$ es la covarianza entre la velocidad vertical del viento y la humedad absoluta. El calor almacenado en la superficie $(Q s)$ fue calculado por residuo en la ecuación 1.

También se hizo el cálculo del área fuente para conocer la representatividad espacial de los sensores en el bosque urbano, es decir, estimar qué fracción las mediciones corresponde al bosque o a la urbe. Para el bosque natural no se consideró necesario este cálculo, ya que la cobertura del suelo es homogénea al menos en $3 \mathrm{~km}$ a la redonda. Se utilizó el modelo de Schuepp et al., (1990), dado por la ecuación:

$$
f(x)=\frac{1}{Q o} \frac{d Q}{Q x}=\frac{2 X_{m}}{x^{2}} \phi_{m} \exp \left[\frac{-2 X_{m}}{x} \phi_{m}\right]
$$

donde $x$ es la distancia desde el punto de medición (metros), $X_{m}$ es la distancia a la cual el área fuente tiene un máximo (metros), y $\phi_{m}$ es una función de corrección de la estabilidad que puede expresarse como (Dyer, 1974):

$$
\phi_{m}=\left[1-16 \frac{Z}{L}\right]^{-0,25}
$$


$z$ es la altura de medición (metros) y $L$ es la longitud de Monin-Obukhov (metros, Monin \& Obukhov, 1954) obtenida con la ecuación:

$$
L=\frac{u^{* 3}}{k \frac{g Q h}{T a \rho C p}}
$$

donde

$$
\frac{Q h}{\rho C p}=\overline{w^{\prime} T a^{\prime}}
$$

Como se mencionó antes, $\overline{W^{\prime} T a^{\prime}}$ es la covarianza entre la velocidad vertical del viento y la temperatura del aire. $u^{*}$ es la velocidad de fricción obtenida mediante la ecuación:

$$
u^{*}=\left[\overline{\left(u^{\prime} w^{\prime}\right)^{2}}+{\overline{\left(v^{\prime} w^{\prime}\right)^{2}}}^{1 / 4}\right.
$$

Por último, $X_{m}$ fue obtenida mediante la ecuación:

$$
X_{m}=\frac{u z}{u^{*} 2 k}
$$

donde $u$ es la velocidad horizontal del viento (m) y $k$ la constante de Von Karman $(0,41)$.

\section{RESULTADOS}

El análisis de área fuente muestra que, durante el día, los sensores obtienen datos tanto del bosque urbano (80\%) como de la ciudad (20\%). En la noche, la contribución cambia a un $20 \%$ bosque y $80 \%$ ciudad.

En el transcurso del día, el bosque de niebla natural presenta valores medios de $367,67 \mathrm{~W} / \mathrm{m}^{2}$ de radiación neta, de los cuales $168.86 \mathrm{~W} / \mathrm{m}^{2}(46 \%)$ son disipados por el flujo de calor sensible, $94,52 \mathrm{~W} / \mathrm{m}^{2}$ (26\%) por el flujo de calor latente y los $104,29 \mathrm{~W} / \mathrm{m}^{2}$ (28\%) restantes fluyen en la superficie (almacenaje). El bosque urbano presenta un aumento de la radiación neta de $35,1 \mathrm{~W} / \mathrm{m}^{2}$ y 16,5 y $24,85 \mathrm{~W} / \mathrm{m}^{2}$ en los flujos de calor sensible y latente respectivamente. El flujo de calor en la superficie sufre una disminución de $6,25 \mathrm{~W} / \mathrm{m}^{2}$. Estas diferencias en los flujos provocan un aumento de la temperatura del aire $\left(17,74\right.$ en el natural $v s .18,17^{\circ} \mathrm{C}$ en el urbano) y de la evapotranspiración (1,39 en el natural vs. 1,63 mm en el urbano). Los datos medios se presentan en la tabla 1 .

\begin{tabular}{|c|c|c|c|c|c|c|c|c|}
\hline Periodo & Sitio & $\boldsymbol{T}\left({ }^{\circ} \mathbf{C}\right)$ & $\begin{array}{c}\text { Evaporación } \\
(\mathbf{m m})\end{array}$ & $\boldsymbol{Q h}$ & $\boldsymbol{Q e}$ & $\boldsymbol{Q s}$ & $\boldsymbol{Q}^{*}$ & $\boldsymbol{\beta}$ \\
\hline \multirow{2}{*}{ Diurno } & Urbano & 18,17 & 1,63 & 185,36 & 119,37 & 98,04 & 402,77 & 1,55 \\
\cline { 2 - 9 } & Natural & 17,74 & 1,39 & 168,68 & 94,52 & 104,47 & 367,67 & 1,78 \\
\hline \multirow{2}{*}{ Nocturno } & Urbano & 14,58 & 0,11 & $-4,91$ & 3,65 & $-58,18$ & $-56,92$ & $-1,34$ \\
\cline { 2 - 9 } & Natural & 14,69 & 0,08 & $-3,85$ & 3,37 & $-50,36$ & $-49,88$ & $-1,14$ \\
\hline
\end{tabular}

Tabla 1. Valores medios de los días despejados durante la temporada seca. 
Durante la noche, la principal fuente de energía es la superficie ya que reemite la almacenada durante el día. En el bosque natural, a pesar de que la superficie almacena más energía que la urbana, para la noche presenta un menor flujo de energía superficial $\left(50,26\right.$ vs. $\left.61,22 \mathrm{~W} / \mathrm{m}^{2}\right)$. De ésta, 49,88 y $56,92 \mathrm{~W} / \mathrm{m}^{2}$ son reemitidos por radiación en el bosque natural y bosque urbano respectivamente.
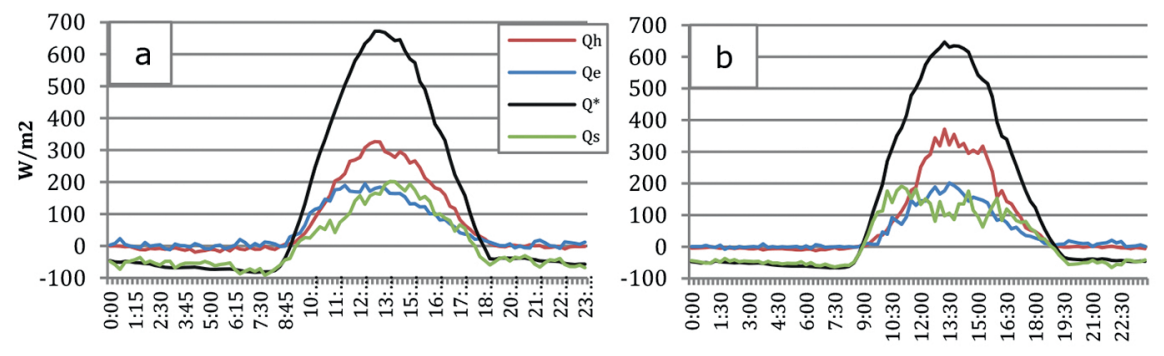

Fig. 2. Comportamiento medio del balance de energía durante temporada seca. a) Bosque Urbano y b)Bosque natural.

Como se puede observar en el comportamiento medio del balance de energía (Fig. 2), las principales diferencias entre sitios se encuentran entre las 9 y las 13 horas (tiempo local; GTM-6). Durante este periodo la diferencia de los flujos de calor sensible y latente son de hasta 60 y $80 \mathrm{~W} / \mathrm{m}^{2}$ respectivamente, presentando los valores más altos en el bosque urbano. Por esto, se presenta un mayor de calentamiento y evapotranspiración en este bosque (Fig. 3).
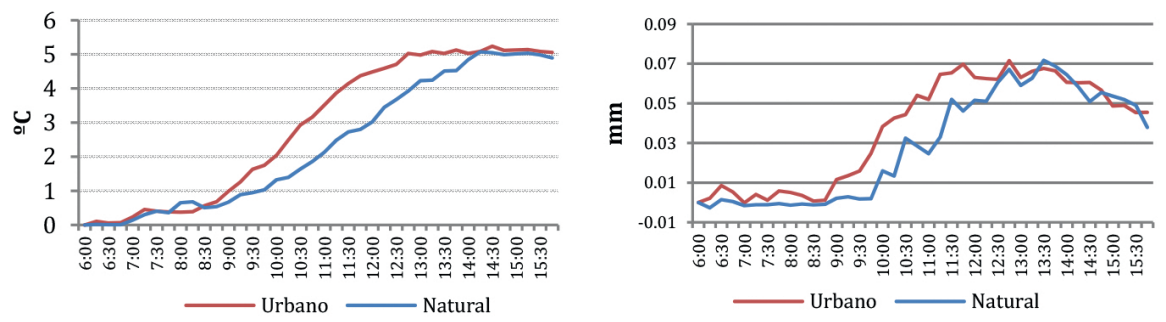

Fig. 3. Calentamiento (izquierda) y evapotranspiración (derecha) acumulados desde las 6 y 16 horas.

Cabe mencionar que el bosque urbano presenta un albedo mayor que el bosque natural $(0,5 v s .0,31)$, por lo que este último absorbe $20 \%$ más de radiación de onda corta alrededor de las 8:30 horas, mientras que para las 13 horas esta diferencia es mínima e inclusive nula $(0,001)$.

En cuanto a la radiación de onda larga, la superficie del bosque natural absorbe (en el día) y reemite (en la noche) mayor cantidad de energía en comparación con el urbano. La radiación reemitida por la atmósfera (radiación de onda larga proveniente de la atmósfera) es mayor en el bosque urbano (diferencia media de $11,91 \mathrm{~W} / \mathrm{m}^{2}$ ) durante todo el día (Fig.4). 

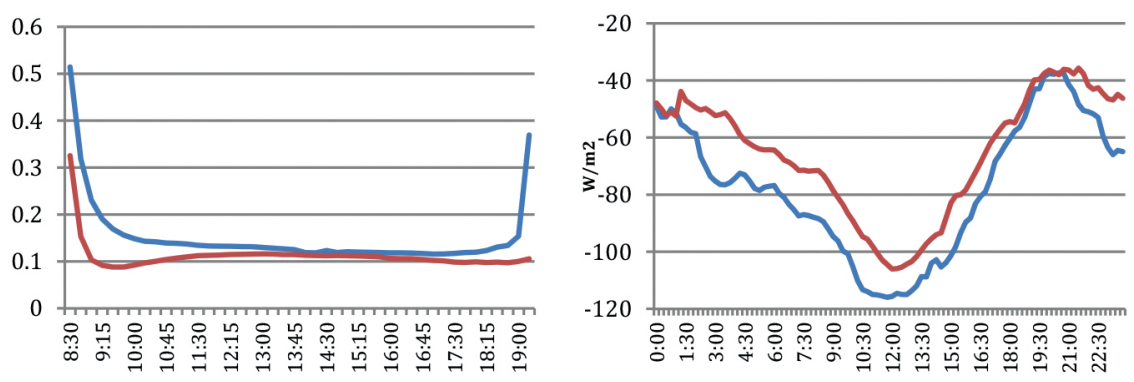

Fig. 4. Albedo (izquierda) y radiación de onda larga incidente (derecha). El color azul indica el bosque urbano y el rojo el natural.

\section{DISCUSIÓN}

El bosque de niebla urbanizado presenta un comportamiento parecido a zonas suburbanas aunque en otro tipo de ecosistema (Barradas et al., 1999; Tejeda-Martínez \& Jáuregui-Ostos, 2005; Cleugh \& Oke, 1986). El bosque natural se comporta similarmente a lo reportado por Giambelluca et al. (2009) para un bosque de niebla en Hawái.

El aumento en los flujos de energía en el bosque urbano se puede atribuir a la ciudad, ya que este bosque se encuentra en el centro geográfico de la urbe y las mediciones durante el día corresponden en un $20 \%$ a suelo urbano. Puede deberse a que la superficie en el bosque urbano recibe más radiación de onda larga durante el día, que posiblemente se pueda explicar por una mayor concentración de gases de efecto invernadero en la ciudad.

Lo anterior podría estar aumentando la capacidad térmica del aire ocasionando un mayor flujo de calor sensible y por ende, un aumento de temperatura (Weng, 2001; Oke, 1982). Esto se puede observar en el calentamiento acumulado (Fig. 3) ya que a medio día el bosque natural tuvo un aumento de casi $5{ }^{\circ} \mathrm{C}$ mientras que el natural a esta misma hora presentó $3^{\circ} \mathrm{C}$. Este calentamiento coincide con las característica de las ciudades, ya que estas diferencia térmicas corresponden con el calentamiento propio del medio urbano, ya que suelen tener mayor temperatura en comparación con sus alrededores.

El aumento del flujo de calor latente en el bosque urbano, es atribuido a la mayor disponibilidad de agua que tiene la ciudad (e.g. riego de jardines). Esto se traduce en un aumento en la evapotranspiración diurna de $0,24 \mathrm{~mm}$ y nocturna de $0.03 \mathrm{~mm}$ por efecto de la ciudad. Por ser una temporada seca, los valores del flujo de calor sensible fueron más altos que los del latente $(\mathrm{Qh}>\mathrm{Qe})$ indicando que la mayor porción de energía disponible es disipada por el primero (Razón de Bowen; $\beta=1,55$ en el urbano y 1,78 en el natural).

Por otra parte, la urbanización provoca un aumento en el albedo superficial (por el concreto de casas y calles) por lo que menos radiación de onda corta podría estarse almacenando, lo cual se refleja en el flujo de calor en la superficie, el cual presenta una reducción $\left(6,43 \mathrm{~W} / \mathrm{m}^{2}\right)$ en el bosque urbano en comparación con el natural. 


\section{AGRADECIMIENTOS}

Este trabajo es parte del proyecto CONACyT-UV (CB2012-183040) "Interacción superficielatmósfera en la zona montañosa central de la vertiente del golfo de México: observaciones y modelación a alta resolución". Agradecemos al Patronato para el Parque Ecológico Cerro de Macuiltépetl por los permisos para la instalación de instrumental y al Consejo Nacional de Ciencia y Tecnología de México (CONACyT) por el apoyo con la beca número 362813. Así mismo, agradecemos al Mtro. Antonio Luna Díaz Peón y a Adrián Álvarez Pérez por su apoyo.

\section{REFERENCIAS}

Aguilar, R. (1996). Recomendaciones y propuestas para el manejo del Parque Ecológico Macuiltépetl. Xalapa, Veracruz: Pronatura Veracruz y Patronato para el Parque Ecológico Macuiltépetl, 510-518.

Baldocchi, D., Hicks, B. and Meyers, T. (1988). Measuring biosphere-atmosphere exchanges of biologically related gases with micrometeorological methods. Ecology 69, 1331-1340.

Barradas, V. L., Tejeda-Martínez, A. and Jáuregui, E. (1999). Energy balance measurements in a suburban vegetated area in Mexico City. Atmospheric Environment 33:4109-4113.

Bubb, P., May, I., Miles, L. and Sayer, J. (2004). Cloud forest agenda. United Kindom: UNEP World Conservation Monitoring Centre.

Burba, G. (2013). Eddy covariance method for scientific, industrial, agricultural, and regulatory applications: a field book on measuring ecosystem gas exchange and areal emission rates. Lincoln, Nebraska: LI-COR Biosciences, ISBN 978-0-61576827-4.

Campos, A. (2006). Response of soil surface CO2-C flux to land use changes in a tropical cloud forest in Mexico. Forest Ecology and Management 234:305-312.

Cleugh, H. A., and Oke, T. R. (1986). Suburban-rural energy balance comparisons in summer for Vancouver, BC. Boundary-Layer Meteorology 36:351-369.

Christen, A., and R. Vogt. (2004). Energy and radiation balance of a central European city. International Journal of Climatology 24:1395-1421.

Dyer, A. J. (1974). A review of flux profile relationships. Boundary-layer Meteorol. 7, 363-372.

Foken, T., and Nappo, C. J. (2008). Micrometeorology. Berlin: Springer. ISBN:9783-54074666-9.

Giambelluca, T., Martin, R., Asner, G., Huang, M., Mudd, R., Nullet, M., Delay, J. and Foote, D. (2009). Evapotranspiration and energy balance of native wet montane cloud forest in Hawai 'i. Agricultural and Forest Meteorology 149:230-243.

INEGI. (2010). Censo de Población y vivienda 2010. México. http://www.inegi.org. $\mathrm{mx} / \mathrm{est} / \mathrm{contenidos/proyectos/ccpv/cpv2010/Default.aspx.}$

Monteith, J.L. and Unsworth, M.H. (2008). Principles of Environmental Sciences. Amsterdam: Elsevier. 
Monin, A. S., and Obukhov, A. (1954). Basic laws of turbulent mixing in the surface layer of the atmosphere. Contrib. Geophys. Inst. Acad. Sci. USSR 151:163-187.

Moreno, M.C., Jáuregui E. and Tejeda-Martínez, A. (2002). Measurements of surfaceatmosphere energy balance components in central Barcelona (Spain)during summer. Col. Asoc. Geógrafos Españoles, No. 60: 7-18.

Oke, T. R. (2002). Boundary Layer Climates. Routledge. ISBN:0-203-4071545-4.

Oke, T. R., Spronken-Smith, R. A., Jauregui, E. and Grimmond, S. (1999). The energy balance of central Mexico City during the dry season. Atmospheric Environment 33:3919-3930.

Oke, T. R. (1982). The Energetic Basis of the Urban Heat Island. Quart. J. Roy. Meteorol. Sot. 108, 1-24. Extended Abstracts of an Expert Meeting, Antwerp, Belgium, April 2000, EUR 19447, European Commission.

Schönwiese, C. (1994). Klimatologie. Stuttgart: Verlag Eugen Ulmer. Stuttgart, 350p.

Schuepp, P.H., Leclerc, M.Y., MacPherson, J.I., Desjardins, R.L. (1990). Footprint prediction of scalar fluxes from analytical solutions of the diffusion equation. Boundary-Layer Meteorol 50:355-373.

SMN, Servicio Meteorológico Nacional (2016). Información Climatológica. http:// smn.cna.gob.mx/es/informacion-climatologica-ver-estado?estado=ver

Stadtmuller, T. (1987). Cloud Forests in the Humid Tropics. A bibliographic review. Costa Rica: United Nations University, Tokyo and CATIE, Turrialba,.

Tejeda-Martínez, A., García-Cueto, O. R., Aquino-Martínez, L. P., Nava-Assad, M. (2016). Simple statistical models of surfacelatmosphere energy fluxes and their hysteresis in a desertic Mexican city (Mexicali). Atmósfera 29:129-139.

Tejeda-Martínez, A. and Jáuregui-Ostos, E., (2005). Surface energy balance measurements in Mexico City region: a review. Atmósfera, 18: 1-23.

United Nations. (2015). World Urbanization Prospects: The 2014 Revision. ISBN 978-92-1-151517-6.

Weng Q. (2001). A remote sensing GIS evaluation of urban expansion and its impact on surface temperature in the Zhujiang Delta, China. Int J Remote Sens 22:19992014

Williams-Linera, G. (2012). El bosque de niebla del centro de Veracruz: ecología, historia y destino en tiempos de fragmentación y cambio climático. Xalapa, México: Inecol.

Williams-Linera, G., Robert, H., and Vera, M. (2002). La fragmentación del bosque mesófilo de montaña. Madera y bosques 8:73-89. 